\title{
BMJ Open Effect of the type of maternal pushing during the second stage of labour on obstetric and neonatal outcome: a multicentre randomised trial-the EOLE study protocol
}

\author{
Chloé Barasinski, ${ }^{1,2}$ Françoise Vendittelli ${ }^{1,2,3}$
}

To cite: Barasinski C, Vendittelli F. Effect of the type of maternal pushing during the second stage of labour on obstetric and neonatal outcome: a multicentre randomised trial一 the EOLE study protocol. BMJ Open 2016;6:e012290. doi:10.1136/bmjopen-2016012290

- Prepublication history and additional material is available. To view please visit the journal (http://dx.doi.org/ 10.1136/bmjopen-2016012290)

Received 13 April 2016 Revised 24 October 2016 Accepted 8 November 2016

CrossMark

For numbered affiliations see end of article.

\section{Correspondence to} Chloé Barasinski; cbarasinski@chuclermontferrand.fr

\section{ABSTRACT}

Introduction: The scientific data currently available do not allow any definitive conclusion to be reached about what type of pushing should be recommended to women during the second stage of labour. The objective of this trial is to assess and compare the effectiveness of directed open-glottis pushing versus directed closed-glottis pushing. Secondary objectives are to assess, according to the type of pushing: immediate maternal and neonatal morbidity, intermediate-term maternal pelvic floor morbidity, uncomplicated birth, and women's satisfaction at 4 weeks post partum.

Methods and analysis: This multicentre randomised clinical trial compares directed closed-glottis pushing (Valsalva) versus directed open-glottis pushing during the second stage of labour in 4 hospitals of France. The study population includes pregnant women who received instruction in both types of pushing, have no previous caesarean delivery, are at term and have a vaginal delivery planned. Randomisation takes place during labour once cervical dilation $\geq 7 \mathrm{~cm}$. The principal end point is assessed by a composite criterion: spontaneous delivery without perineal lesion (no episiotomy or spontaneous second-degree, third-degree or fourth-degree lacerations). We will need to recruit 125 women per group. The primary analysis will be by intention-to-treat analysis, with the principal results reported as crude relative risks (RRs) with their $95 \% \mathrm{Cls}$. A multivariate analysis will be performed to take prognostic and confounding factors into account to obtain adjusted RRs.

Ethics and dissemination: This study was approved by a French Institutional Review Board (Comité de Protection des Personnes Sud Est 6:NAU1168). Results will be reported in peer-reviewed journals and at scientific meetings. This study will make it possible to assess the effectiveness of 2 types of directed pushing used in French practice and to assess their potential maternal, fetal and neonatal effects. Findings from the study will be useful for counselling pregnant women before and during labour.

Trial registration number: Agence national de sécurité du médicament et des produits de santé (ANSM): 150099B-22 and IDRCB: 2014-A01920-47. ClinicalTrials. gov: NCT02474745. Pre-result stage.

\section{Strengths and limitations of this study}

- The EOLE study is designed as a randomised controlled, non-blinded multicentre superiority trial with two parallel groups, comparing directed closed-glottis pushing to directed open-glottis pushing during the active phase of the second stage of labour.

- To be sure that the intervention is standardised in all participating centres, both the midwives-investigators involved and the women will receive specific instruction in these breathing techniques (separate videos for professionals and pregnant women).

- The co-interventions (analgesia, maternal position, oxytocin use to augment labour, etc) associated with labour and its monitoring will be identical to the usual management in the participating maternity units.

- Compliance will be monitored by each investigator for each randomised woman (number of contractions, type of pushing for each contraction, number of pushes per contraction).

- Loss to follow-up can be anticipated for one of our secondary end points: negative effect of labour on the pelvic floor and urinary function (Pelvic Organ Prolapse Quantification System (POP-Q) and International Consultation on Incontinence modular Questionnaire-Short Form (ICIQ-SF)), which will be assessed at the postpartum visit (between 6 and 8 weeks after delivery).

\section{INTRODUCTION}

This EOLE protocol followed published guidelines for clinical trials protocols, along with the Standard Protocol Items: Recommendations for Interventional Trials (SPIRIT 2013 Checklist). This protocol is V.03, written on 28 April 2015. The study began in July 2015 after all authorisations 
necessary under French law were granted. See the trial registration data for all registration details (table 1). The literature review and study justification are presented in the Background and significance section.

\section{BACKGROUND AND SIGNIFICANCE}

There are two types of pushing during the second stage of labour: open or closed glottis. Each can be either directed (ie, coached) or spontaneous (left to the woman's initiative). Although neither closed-glottis nor open-glottis pushing is a natural or spontaneous type of pushing (except when vomiting), the practice of French professionals managing deliveries appears oriented towards the use of directed pushing, especially directed closed-glottis pushing.

Closed-glottis pushing is also called Valsalva pushing. It is based on high abdominal pressure induced by descent of the diaphragm, which acts like a piston on the uterus and helps the mobilisation of the descending fetus by compressing the uterine fundus. ${ }^{1}$ The uterus is projected downwards and forward, without any counterpressure, because the rectus abdominis muscles spread out. ${ }^{2}$ During delivery, this mode of pushing can lead to various changes in the fetomaternal circulation. After forced inspiration, the reduction in the maternal blood pressure may lead to a reduction in placental perfusion and therefore also in fetal oxygenation. One would then observe a reduction in $\mathrm{pH}$ and partial pressure of oxygen $\left(\mathrm{pO}_{2}\right)$ at the level of the umbilical artery. ${ }^{3}$ This pushing technique could therefore, in theory, promote the onset of hypoxia and therefore fetal acidosis. ${ }^{2}$ This type of pushing may also have harmful consequences on pelvic floor function. That is, during these pushing efforts, the diaphragm is projected downwards, bringing with it the organs. This pushing may thus promote the factors that cause prolapse, thus leading to urinary incontinence, pelvic floor problems and eventually prolapse. Moreover, the high abdominal pressure accompanied by descent of the organs causes pressure on the perineum which, in response to the myotatic reflex, bulges and contracts. Even more powerful expulsive efforts are then required to expel the descending fetus, thereby stretching the lower birth canal. ${ }^{4}$

Open-glottis pushing, also called slow exhalation through pursed lips, involves different muscular mechanisms aimed at reproducing expulsive reflexes, but without their power. Here, it is the abdominal muscles that contract: the transverse and oblique muscles by compressing the uterus on both sides allow the descending fetus to progress. $^{2}$ In contrast to closed-glottis pushing, the diaphragm rises. There is therefore no organ descent to be pulled along with the uterus, no perineal contraction, and therefore no perineal muscle resistance. This pushing should therefore not promote prolapse (or at least it will do so less). During this pushing, the parturient is not holding her breath, nor is her diaphragm under high pressure. Theoretically, therefore, fewer haemodynamic modifications should occur.
We have found seven single-centre randomised trials that studied obstetric and neonatal outcome in comparing open-glottis pushing, directed or spontaneous, with directed closed-glottis pushing; they have been reported in nine published articles, ${ }^{5-13}$ and two meta-analyses. ${ }^{14} 15$ The intervention studied varied between studies: although the control groups always used directed Valsalva pushing, the open-glottis breathing in the intervention group was sometimes spontaneous ${ }^{7-10} 1213$ and sometimes directed. ${ }^{5} 611$ The meta-analysis by Prins et $\mathrm{al}^{14}$ compared self-directed spontaneous pushing with directed Valsalva pushing $(n=425)$ and concluded that closed-glottis pushing had a negative effect on urodynamic prognosis, consistent with the findings by Schaffer $e t$ al..$^{9}$ They underlined, in conclusion, the need for other studies on this subject. Lemos $e t a l^{15}$ published a second meta-analysis in the Cochrane database, comparing spontaneous (in practice, non-directed openglottis) versus directed (in practice, closed-glottis) pushing. The authors included seven studies, but the subanalyses of maternal and fetal comorbidities were based on only one to three studies. They found no differences for any of the criteria studied, except for the duration of expulsive efforts, which appeared shorter in the spontaneous pushing group (mean difference $-5.20 \mathrm{~min}$; $95 \%$ CI -7.78 to $-2.62, \mathrm{n}=100$ ). They also concluded that randomised trials of good quality are necessary for the reliable determination of the possible good and harmful effects of these different techniques.

When we limit our review to studies between 'coached Valsalva' pushing versus 'coached open-glottis' pushing, the practices used in France, we note that the study inclusion criteria included a gestational age of at least 36 weeks or more, cephalic presentation and a singleton pregnancy. ${ }^{5} 611$ Only one trial included multiparous women. ${ }^{6} \mathrm{~A}$ recent review points out that co-interventions varied between studies, ${ }^{16}$ as did the mother's position during the second stage of labour. One study left position to the woman's choice $;^{6}$ two others mandated a specific position: dorsal decubitus position in one ${ }^{11}$ and semirecumbent in the other. ${ }^{5}$ Globally, the quality of the randomisation was not good. ${ }^{16}$ One study reported using sealed envelopes, ${ }^{6}$ one failed to specify the kind of envelope used, ${ }^{11}$ and the last one did not specify the mode of randomisation. ${ }^{5}$ The moment of randomisation varied between studies, except for the study that did not describe it. ${ }^{5}$ Compliance with the allocated intervention was not described in one study ${ }^{11}$ and varied in the others from $34.4 \%$ to $100 \% .^{5}{ }^{6}$ Two studies did not use intention-to-treat analysis. ${ }^{6}{ }^{11}$ Another study did not adjust its results for the children's birth weights, which were higher in the closed-glottis group. ${ }^{6}$ Postrandomisation exclusions were observed in one study. ${ }^{6}$ Moreover, the outcomes measured varied from study to study. ${ }^{16}$

The objective of our study is to assess and compare the effectiveness of directed closed-glottis (Valsalva) pushing (pushing while holding one's breath) versus directed 
Table 1 Trial registration data for the EOLE study

\begin{tabular}{l} 
Data category \\
\hline Primary registry and trial \\
identifying number \\
Date of registration in primary \\
registry \\
Secondary identifying numbers \\
Source(s) of monetary or \\
material support \\
Primary sponsor \\
Secondary sponsor(s) \\
Contact for public queries \\
Contact for scientific queries
\end{tabular}

Information

ClinicalTrials.gov

NCT02474745

5 June 2015

AU 1168, 2014-A01920-47, 150099B-22

French Ministry of Health (grant number PHRC 2005 05.09)

Délégation de la Recherche Clinique, Centre Hospitalier Universitaire de Clermont-Ferrand NA

cbarasinski@chu-clermontferrand.fr

cbarasinski@chu-clermontferrand.fr

fvendittelli@chu-clermontferrand.fr

Public title

Scientific title

Countries of recruitment

Health condition(s) or problem

(s) studied

Intervention(s)

Key inclusion and exclusion

criteria

Effect of the type of maternal pushing during the second stage of labour on obstetric and neonatal outcome: a multicentre randomised trial-the EOLE Study

Effect of the type of maternal pushing during the second stage of labour on obstetric and neonatal outcome: a multicentre randomised trial-the EOLE Study

France

Delivery, 2nd stage of labour

Intervention group: Directed open-glottis pushing

Control group: Directed closed-glottis pushing

Ages eligible for study: $\geq 18$ years

Sexes eligible for study: Female

Accepts healthy volunteers: Yes

Inclusion criteria: Women who are nulliparous or multiparous ( $\geq 1$ previous child), who attended a complete training session about the types of pushing assessed in this trial during prenatal childbirth preparation and parenthood classes (regardless of the type of prenatal preparation), for whom a vaginal delivery was planned at the end of pregnancy, admitted to the maternity ward between 37 and 42 weeks of gestation ( $\geq 37$ and $\leq 42$ weeks) in spontaneous or induced labour, with cervical dilation $\geq 7 \mathrm{~cm}$, with a singleton pregnancy in cephalic presentation, who provided informed consent in writing and who speaks and writes French.

Exclusion criteria: Minors or adults incapable of providing consent for the study or with a disorder contraindicating prolonged pushing or with a uterine scar (previous caesarean or other surgery) or with a contraindication to vaginal delivery or a maternal disease that could justify in termination of the pregnancy (hemolysis, elevated liver enzymes and low platelets count syndrome, pre-eclampsia (hypertension with albuminuria $>0.3 \mathrm{~g}$ per 24 hours), eclampsia, abruptio placentae, etc) or with a major genital haemorrhage or a major fetal malformation and/or hydramnios or oligohydramnios, and/or fetal growth restriction $(<5$ th centile) diagnosed in utero, or with a fetal heart rate anomaly before randomisation or in utero fetal death or multiple pregnancy.

Study type Interventional

Randomised, controlled, non-blinded multicentre superiority trial with 2 parallel groups July 2015

250

\section{Currently recruiting}

Composite criterion: spontaneous delivery without perineal lesion (episiotomy, spontaneous second-degree, third-degree or fourth-degree lacerations)

Occurrence of perineal lesions such as episiotomy or a severe perineal laceration

Immediate postpartum haemorrhage

Effect on pelvic floor and urinary function (POP-Q and ICIQ-SF)

Women's satisfaction, assessed by a validated Swiss questionnaire

ICIQ-SF, International Consultation on Incontinence modular Questionnaire-Short Form; NA, not applicable; POP-Q, Pelvic Organ Prolapse Quantification System.

open-glottis pushing (pushing during a prolonged exhalation), during the second stage of labour. The primary outcome is a composite criterion: spontaneous delivery with no perineal lesion (ie, no episiotomy or seconddegree, third-degree or fourth-degree lacerations).
Secondary objectives are to assess the rates of: (1) immediate maternal morbidity, (2) intermediate-term maternal pelvic floor morbidity, (3) uncomplicated birth, (4) immediate neonatal morbidity and (5) women's satisfaction at 4 weeks post partum. 


\section{METHODS AND ANALYSIS}

\section{Study design}

The EOLE study is designed as a randomised, controlled, non-blinded multicentre superiority trial with two parallel groups, comparing directed closed-glottis pushing with directed open-glottis pushing during the active phase of the second stage of labour. Randomisation is being performed with a 1:1 allocation.

\section{Study setting}

There are four centres, all in France: two university hospitals and two general hospitals.

\section{Eligibility criteria}

Women of any parity are eligible for inclusion if they: have taken an antenatal class that includes training in the types of pushing, have planned a vaginal delivery, are admitted at 37-42 weeks of gestation for spontaneous or induction of labour, with cervical dilation $\geq 7 \mathrm{~cm}$, a singleton pregnancy in cephalic presentation, and provide written consent, and speak and write French. Women will be excluded if they are younger than 18 years, have had a previous caesarean delivery or other uterine surgery (that leaves a scar), have a disease contraindicating expulsive efforts or that may justify emergency delivery (HELLP syndrome, preeclampsia, abruptio placentae, etc), or severe genital haemorrhage, or major fetal malformation and/or hydramnios or oligohydramnios, and/or intrauterine growth restriction diagnosed in utero (ie, below the 5th centile for gestational age and sex), or a fetal heart rate anomaly or an in utero fetal death before randomisation.

\section{Interventions}

In the intervention group, directed open-glottis pushing (with prolonged exhalation) must be explained to the women and professionals as follows: 'After inhaling deeply, the woman will exhale while pulling in her stomach in such a way that she can use the contraction of her abdominal muscles to help the fetus descend through the birth canal. She should push as long as possible'.

In the control group, directed closed-glottis pushing (pushing while holding one's breath) should be explained to the women and professionals as follows: 'After inhaling deeply, the woman should push very hard downwards to the perineum, while holding the inhaled breath in her lungs. She should push as hard and as long as possible'.

The pregnant women receive information about the study and instruction about the types of pushing between 29 and 37 weeks of gestation. To be sure that the intervention is standardised in all participating centres, the women receive specific instruction in these breathing techniques. During one session of antenatal classes, pregnant women see a video specifically created for them, describing and illustrating both types of directed pushing. Those who have completed this instruction receive a card attesting to this instruction, which they are asked to keep with their blood group cards (to minimise the possibility of losing it).

Both techniques are coached by the midwife managing the delivery. This study is not assessing spontaneous pushing. Women in both groups are coached to push three times per contraction, as usual in France, if possible. Elsewhere, all participating staff-that is, all professionals teaching antenatal classes who agree to support the study and the midwives-investigators who include the women and then manage the delivery-are trained in advance in both pushing techniques so that they can teach and support the women. A video intended specifically for professionals has been produced for the study to standardise the information provided to patients.

\section{Co-interventions}

The co-interventions (analgesia, maternal position, use of oxytocin to augment labour, etc) associated with labour and its monitoring are identical to the usual management in the participating maternity units. The midwife managing the delivery determines the moment that active labour and thus bearing down and pushing efforts begin. The fetal heart rate and the frequency of uterine contractions are monitored continuously, after randomisation, with an external tocodynamometer, throughout labour and expulsive efforts.

Investigators who determine after 20 min that the type of pushing used appears ineffective can ask mothers to switch to the other type, if they think it useful. In the latter case, the women will remain in their initial group. If fetal heart rate abnormalities or other obstetric emergencies occur, the midwife and/or the supervising obstetrician will be the sole decision-makers, jointly with the mother, to the extent possible, for the ensuing medical management (change in pushing technique, instrumental or caesarean delivery).

\section{Outcomes}

The principal end point is a composite criterion: spontaneous delivery with no perineal lesion (no episiotomy or second-degree, third-degree or fourth-degree lacerations).

Our secondary outcome measures are divided into three categories. For the mother, we will assess (1) immediate maternal morbidity, defined by the occurrence of perineal lesions, such as episiotomy or a severe perineal laceration (third or fourth degree) or immediate postpartum haemorrhage (blood loss $>500 \mathrm{~mL}$ in the 24 hours after delivery); (2) intermediate-term maternal morbidity will be defined by effect on pelvic floor and urinary function (Pelvic Organ Prolapse Quantification System (POP-Q) and International Consultation on Incontinence modular QuestionnaireShort Form (ICIQ-SF)) at 2 months after delivery or at the postpartum visit, ${ }^{17} 18$ and (3) women's satisfaction at 
4 weeks post partum (Questionnaire d'Evaluation du Vécu de l'Accouchement questionnaire). Secondary neonatal outcomes will be immediate neonatal morbidity defined by a 5 -min Apgar score $<7$ or an umbilical artery $\mathrm{pH}<7.10$ or the need for resuscitation in the delivery room (defined by one or more of the following events: aspiration by a laryngoscope, mask ventilation, oxygenation by a nasal cannula, or hood mask, tracheal intubation, endotracheal ventilation or cardiac massage) or transfer to a neonatology department. Finally, for both mother and child, a composite end point which might be considered a quality indicator, is uncomplicated birth, defined by birth without caesarean, operative intervention or obstetrical manoeuvres, without postpartum haemorrhage $(500 \mathrm{~mL})$, without seconddegree, third-degree or fourth-degree perineal lacerations, and with a 5 min Apgar $\geq 9$.

\section{Participant timeline}

The study schedule is described in the schedule (figure 1).

\section{Sample size}

For $\alpha=0.05$ and a power of $90 \%$, based on data from the Audipog database (a French national database: Audipog: http://www.audipog.net/interro-choices.php) that women who deliver spontaneously, without any perineal lesion (ie, without episiotomy or spontaneous seconddegree, third-degree or fourth-degree lacerations) account for $49.6 \%$ of all parturients, the investigators estimate that the use of a two-sided test to show an absolute difference between groups of $20 \%$ (or $49.6 \%$ vs $69.6 \%$, a relative difference of the order of $40 \%$ ) would require 125 women per group.

\section{Recruitment}

Again using information from the Audipog database ( $n=413888$ women), we estimate that $63.4 \%$ of women give birth without a planned caesarean to a singleton fetus at a term $\geq 37$ weeks of gestation and have neither a uterine scar nor a utero fetal death. Among these women, $40 \%$ take a childbirth and parenting preparation class. Accordingly, 25\% of the women in each maternity ward should be eligible to participate. The four participating maternity units account for 8411 deliveries per year and should thus have 2103 eligible women each year. We estimate that $70 \%$ of the women-1472will agree to participate each year. Although it may be feasible to complete the study in 1-year, we have planned for it to last 2 years. To optimise women's participation in this study, information posters are displayed in the healthcare facilities where antenatal care, preparation for delivery and deliveries take place and in the offices of the midwives in private practice supporting the study. Brochures have also been made available to pregnant women. They were also informed about the study during childbirth preparation and parenthood classes and during antenatal care visits with midwives or obstetricians. These obstetric professionals have been made aware of the value of supporting this study during meetings at and outside the hospital.

\section{Allocation and blinding}

A computer program designed by an independent group of the clinical research centre is creating the randomisation list, in blocks of four to six (randomly chosen by the electronic system and well balanced by centre) and stratified by maternity ward and within maternity wards by both parity (nulliparous vs

\begin{tabular}{|c|c|c|c|c|c|c|c|}
\hline & \multirow{3}{*}{$\frac{\text { Training }}{t_{-2}}$} & \multicolumn{6}{|c|}{ STUDY PERIOD } \\
\hline & & \multicolumn{3}{|c|}{ Enrolment \& Allocation } & \multicolumn{3}{|c|}{ Post-allocation } \\
\hline & & $t_{-1}$ & 0 & t1 & t2 & t1 & †2 \\
\hline TIMEPOINT & $\begin{array}{l}29-37 \text { weeks } \\
\text { of gestation }\end{array}$ & $\begin{array}{c}\text { Admission: } 37-42 \\
\text { weeks of gestation }\end{array}$ & Randomisation & Delivery & $\begin{array}{c}\text { Immediate } \\
\text { post-partum }\end{array}$ & $\begin{array}{c}\text { Follow-up } \\
1 \text { month }\end{array}$ & $\begin{array}{l}\text { Follow-up } \\
2 \text { months }\end{array}$ \\
\hline $\begin{array}{l}\text { Training during } \\
\text { antenatal class }\end{array}$ & $x$ & & & & & & \\
\hline \multicolumn{8}{|l|}{ ENROLMENT: } \\
\hline \multirow{2}{*}{$\begin{array}{l}\text { Eligibility screen } \\
\text { Informed consent }\end{array}$} & & $x$ & & & & & \\
\hline & & $x$ & & & & & \\
\hline Allocation & & & $x$ & & & & \\
\hline \multicolumn{8}{|l|}{ INTERVENTIONS: } \\
\hline \multicolumn{8}{|l|}{$\begin{array}{r}\text { Open-glottis pushing } \\
\text { or } \\
\text { Closed-glottis pushing }\end{array}$} \\
\hline \multicolumn{8}{|l|}{ ASSESSMENTS: } \\
\hline Delivery & & & & $x$ & & & \\
\hline $\begin{array}{r}\text { Immediate post- } \\
\text { partum }\end{array}$ & & & & & $x$ & & \\
\hline $\begin{array}{r}\text { Satisfaction } \\
\text { questionnaire }\end{array}$ & & & & & & $x$ & \\
\hline $\begin{array}{r}\text { Assessments of pelvic } \\
\text { floor function }\end{array}$ & & & & & & & $X$ \\
\hline
\end{tabular}

Figure 1 Schedule of enrolment, interventions and assessments of women in the EOLE study. 
multiparous) and epidural analgesia use at randomisation. The randomisation and the data collection will be performed at a website available 24 hours a day.

After the midwives-investigators have verified the inclusion and exclusion criteria and collected the signed informed consent, the randomisation will take place. There is no conceivable way to conduct this study on either a double-blinded or single-blinded basis.

\section{Data collection, management and analysis}

Most of the information required for the study is recorded in women's computerised medical files at each centre; it was thus collected before randomisation. The other data are collected either after delivery by the investigator (compliance, station of the descending fetus at the beginning of expulsive efforts), or at a postnatal visit by a well-trained gynaecologist obstetrician or a midwife to collect the woman's perception of pelvic floor function, determined by a self-administered questionnaire (ICIQ-SF) $^{18}$ and a less subjective assessment with the POP-Q. ${ }^{17}$ All data will be entered in an electronic research case file for each woman.

Women will complete the satisfaction questionnaire directly online a month after delivery, after receiving an email asking them to do so and including a link, personal log-in name and password to enter the website.

The primary analysis will be by intention to treat and a secondary analysis per protocol. The baseline characteristics of the women and children (age, weight, parity, etc), the women's satisfaction and pelvic floor function in the two groups will be compared with a $\chi^{2}$ test (or Fisher's exact test, as appropriate) for the qualitative variables and by Student's t-test for the quantitative variables. The principal results will be reported as crude relative risks (RRs) with their 95\% CIs. Simple descriptive statistics will be used to report some relatively infrequent data (such as transfer to the neonatal intensive care unit (ICU)). A multivariate analysis will be performed to take prognostic and confounding factors (parity, analgesia, maternal position, operative delivery, etc) into account to obtain adjusted RRs. A centre effect will be sought and handled with a Cochran-Mantel Haenszel test, to compare the efficacy of the type of pushing in each group. An analysis according to parity is planned.

An interim analysis will be planned after half of the planned women have been included. Adherence to the type of pushing in France is currently unknown. To preserve an overall threshold of $5 \%$ for the final analysis, the interim analysis will use a threshold of $0.1 \%$.

The missing data will be treated as missing.

\section{Monitoring}

A data monitoring committee met at the study launch and will continue to meet throughout the study, on its own initiative or at the request of the sponsor (Clermont-Ferrand University Hospital Centre). It will also issue a general report about the course of the study procedures and may contribute to decisions in the following circumstances: opinion about early stopping of the study; opinion about significant modifications of the protocol or taking new scientific data into account. This committee is composed of two physicians, two midwives and a biostatistician, all independent of the study investigators and methodological advisors. The trial sponsor has no role in the design of the trial, the collection, analysis or interpretation of the data, or the future writing of the manuscript.

No serious adverse events directly associated with the study intervention are expected. Nonetheless, the following complications must be reported: death or transfer to adult or neonatal ICU. Any serious adverse event in the first 8 weeks post partum must be reported to the principal investigator and the study sponsor within 24 hours by the investigator, on a special form. The institutional review board must also be informed no later than 7 days after the event. The investigator shall make a determination about the existence of any causal relation between the serious adverse event and the study. When a serious adverse event persists at the end of the study, the investigator shall continue the follow-up of the woman until the event is considered resolved.

According to the French Law and the French research quality guideline, an external audit is not requested for this low-risk trial.

\section{ETHICS AND DISSEMINATION \\ Protocol amendments}

Any modifications to the protocol must be characterised as substantial or minor. Substantial modifications must be approved de novo by the Patient Protection Committee and/or the ANSM (National Agency for the Drug and Medical Product Safety).

\section{Consent}

The women will be informed completely and fairly of the objectives and constraints of the study, of any possible risks, of their right to refuse to participate and of their right to withdraw their consent and end their participation at any time. All of this information will be included on the information and consent form provided to the woman (see online supplementary files 1 and 2). The investigator shall collect each woman's free and informed consent in writing.

\section{Confidentiality}

In compliance with French regulations, persons with direct access to the data will take all precautions necessary to ensure the confidentiality of the data concerning women participating in this study. Moreover, the storage of data collected for this study must comply with French guidelines issued by the competent agency (CNIL: National Data Protection Authority-http://www.cnil.fr).

The study documents shall be archived on the premises of the PEPRADE research team until the end of 
their practical utility and then stored in the sponsor's central archives for 15 years.

\section{Access to data}

At the conclusion of the research, the data collected about the participants will be anonymised before the statistical analysis. Access to the anonymised data will be limited to the two principal investigators and to the statisticians accredited by the sponsor.

\section{Dissemination policy}

The data will be divulged only after the joint accord of the principal investigator and the sponsor. The results will be the subject of scientific communications and publications. The authorship eligibility will follow the Recommendations for the Conduct, Reporting, Editing, and Publication of Scholarly Work in Medical Journals, 2015 (http://www.icmje.org).

\section{Author affiliations}

${ }^{1}$ Pôle Femme et Enfant, The Clermont-Ferrand University Hospital, ClermontFerrand, France

${ }^{2}$ EA 4681, PEPRADE (Perinatalogy, pregnancy, Environment, medical care PRActices and DEvelopment), Clermont University, University of Auvergne, Clermont-Ferrand, France

${ }^{3}$ The AUDIPOG Sentinel Network (Association des Utilisateurs de Dossiers informatisés en Pédiatrie, Obstétrique et Gynécologie), RTH Laennec Medical University, Lyon, France

Contributors $\mathrm{CB}$ and FV designed the study, wrote the study protocol and this article, and FV obtained the funding for the study. $\mathrm{CB}$ is the coordinator-investigator and FV the methodologist and supervisor of $\mathrm{CB}$, a PhD student.

Funding This work was supported by the French Ministry of Health (grant number PHRC 2005 05.09).

Competing interests None declared.

Ethics approval The EOLE study protocol $(\mathrm{V} .1,2014)$ was approved by the institutional review board on 21 May 2015 (Patient Protection Committee Southeast VI, AU 1168), and by the ANSM (National Agency for the Drug and Medical Product Safety) on 30 January 2015 (IDRCB: 2014-A01920-47).

Provenance and peer review Not commissioned; externally peer reviewed.

Open Access This is an Open Access article distributed in accordance with the Creative Commons Attribution Non Commercial (CC BY-NC 4.0) license, which permits others to distribute, remix, adapt, build upon this work noncommercially, and license their derivative works on different terms, provided the original work is properly cited and the use is non-commercial. See: http:// creativecommons.org/licenses/by-nc/4.0/

\section{REFERENCES}

1. Calais-Germain B. Le périnée féminin et l'accouchement [The Female Perineum and Childbirth] (in French.). Méolans-Revel: Désiris, 1996.

2. de Gasquet B. Pour une poussée moins traumatique [For less traumatic pushing]. In: Schaal JP, Riethmuller D, Maillet R, et al. Mécaniques \& techniques obstétricales [Obstetrical Mechanics and Techniques ]. 3rd edn. (in French). Montpellier: Sauramps Medical, 2007:289-93.

3. Caldeyro-Barcia R, Giussi G, Storch E, et al. The bearing-down efforts and their effects on fetal heart rate, oxygenation and acid base balance. J Perinat Med 1981;9(Suppl 1):63-7.

4. Shafik A, El-Sibai O, Shafik AA, et al. Effect of straining on perineal muscles and their role in perineal support: identification of the straining-perineal reflex. J Surg Res 2003;112:162-7.

5. Barnett MM, Humenick SS. Infant outcome in relation to second stage labor pushing method. Birth 1982;9:221-9.

6. Parnell C, Langhoff-Roos J, Iversen R, et al. Pushing method in the expulsive phase of labor. A randomized trial. Acta Obstet Gynecol Scand 1993;72:31-5.

7. Thomson AM. Pushing techniques in the second stage of labour. J Adv Nurs 1993;18:171-7.

8. Thomson AM. Maternal behavior during spontaneous and directed pushing in the second stage of labour. J Adv Nurs 1995;22:1027-34.

9. Schaffer JI, Bloom SL, Casey BM, et al. A randomized trial of the effects of coached vs. uncoached maternal pushing during the second stage of labor on postpartum pelvic floor structure and function. Am J Obstet Gynecol 2005;192:1692-6.

10. Bloom SL, Casey BM, Schaffer Jl, et al. A randomized trial of coached versus uncoached maternal pushing during the second stage of labor. Am J Obstet Gynecol 2006;194:10-13.

11. Yildirim G, Beji NK. Effects of pushing techniques in birth on mother and fetus: a randomized study. Birth 2008;35:25-30.

12. Jahdi $\mathrm{F}$, Shahnazari $\mathrm{M}$, Kashanian $\mathrm{M}$, et al. A randomized controlled trial comparing the physiological and directed pushing on the duration of the second stage of labor, the mode of delivery and Apgar score. Int J Nurs Midwifery 2011;3:55-9.

13. Low LK, Miller JM, Guo Y, et al. Spontaneous pushing to prevent postpartum urinary incontinence: a randomized, controlled trial. Int Urogynecol J 2013;24:453-60.

14. Prins M, Boxem J, Lucas $\mathrm{C}$, et al. Effect of spontaneous pushing versus Valsalva pushing in the second stage of labour on mother and fetus: a systematic review of randomised trials. BJOG 2011;118:662-70

15. Lemos A, Amorim MM, Dornelas de Andrade A, et al. Pushing/ bearing down methods for the second stage of labour. Cochrane Database Syst Rev 2015;(10):CD009124.

16. Barasinski C, Lemery D, Vendittelli F. Do maternal pushing techniques during labour affect obstetric or neonatal outcomes? Gynecol Obstet Fertil 2016;44:578-83.

17. Bump RC, Mattiasson A, Bø K, et al. The standardization of terminology of female pelvic organ prolapse and pelvic floor dysfunction. Am J Obstet Gynecol 1996;175:10-17.

18. Avery K, Donovan J, Peters TJ, et al. ICIQ: a brief and robust measure for evaluating the symptoms and impact of urinary incontinence. Neurourol Urodyn 2004;23:322-30. 